\title{
Quality of life and functional status in patients with cancer of the oral cavity and oropharynx: pretreatment values of a prospective study
}

\author{
Pepijn A. Borggreven • Irma M. Verdonck-de Leeuw • \\ Martin J. Muller • Milou L. C. H. Heiligers • \\ Remco de Bree • Neil K. Aaronson • C. René Leemans
}

Received: 28 December 2005 / Accepted: 10 January 2007 / Published online: 2 February 2007

(C) Springer-Verlag 2007

\begin{abstract}
We assessed the pretreatment healthrelated quality of life (HRQOL) and functional status of patients with advanced oral and oropharyngeal cancer. Eighty patients were investigated. HRQOL was assessed by EORTC QLQ-C30/QLQ-H\&N35 questionnaires. Functional status assessment comprised speech and oral function tests. The results revealed a wide range of HRQOL and functional deficits before treatment. HRQOL appeared to be related to some extent to tumor site (patients with oral tumors reported more pain compared to patients with oropharyngeal tumors) and tumor classification (patients with T3-T4 tumors reported more trouble opening the mouth and felt more ill compared to patients with $\mathrm{T} 2$ tumors). Comorbidity appeared to have a major impact. Patients with comorbidity had significantly worse scores on several scales/items on both the EORTC questionnaires. Functional deficits were related to tumor site, classification and comorbidity. Patients with oral cavity tumors (versus oropharyngeal tumors), patients with T3-T4 tumors (versus T2 tumors), and patients with comorbidity (versus without comorbidity) scored significantly worse on several speech and oral function tests. Impaired speech and
\end{abstract}

P. A. Borggreven · I. M. Verdonck-de Leeuw ( $₫)$.

M. L. C. H. Heiligers · R. de Bree · C. R. Leemans

Department of Otolaryngology-Head and Neck Surgery,

VU University Medical Center, P.O. Box 7057,

1007 MB Amsterdam, The Netherlands

e-mail: im.verdonck@vumc.nl

M. J. Muller · N. K. Aaronson

Division of Psychosocial Research and Epidemiology,

The Netherlands Cancer Institute, Amsterdam,

The Netherlands oral function appeared to be clearly related to global quality of life (QLQ-C30) and self-reported speech (QLQ-H\&N35). Many patients with advanced oral and oropharyngeal cancer have compromised HRQOL and functional status before the start of treatment. In addition to tumor site and tumor classification, comorbidity appears to have a major impact on HRQOL and functional status. Knowledge of pretreatment HRQOL and functional status levels is useful for better understanding the impact of treatment on these outcomes over time.

Keywords Head and neck cancer - Quality of life · Functional status $\cdot$ Speech $\cdot$ Microvascular reconstruction

\section{Introduction}

The most important outcome for cancer patients is overall survival. However, the disease and its treatment often have a major impact on health-related quality of life (HRQOL) and functional status in patients with head and neck cancer [1]. Therefore, HRQOL and functional status are important aspects to consider in treatment evaluation. To interpret outcome following treatment, it is necessary to assess HRQOL and functional status following diagnosis, but before the start of treatment [2].

Studies on pretreatment HRQOL [2-5] and functional status $[6,7]$ often include heterogeneous groups of head and neck cancer patients. However, there are substantial differences between patient groups that are related to tumor site and stage [7]. Patients with advanced oral or pharyngeal cancer, for example, often 
have the poorest HRQOL and functional status [5, 8]. Pretreatment HRQOL among patients with oral or oropharyngeal cancer is often compromised, although it tends to be better compared to HRQOL following treatment [9-11]. There is also evidence that functional status, including oral function, speech and swallowing abilities is significantly deteriorated before treatment [12-15]. Comorbidity is another important factor that varies substantially among subpopulations of patients, and can have a significant influence on the choice of initial treatment, the care that patients receive, and on treatment outcomes [16]. Therefore, comorbidity should be taken into consideration evaluating the HRQOL of patients, both at time of diagnosis, and over the course of treatment [17-19].

The primary objective of the present study is to assess pretreatment HRQOL and functional status in relation to tumor site, tumor classification and comorbidity, in a well-defined group of patients with advanced oral and oropharyngeal cancer.

\section{Patients and methods}

\section{Patients}

Between January 1998 and December 2001, 92 consecutive patients diagnosed with stage II-IV oral or oropharyngeal squamous cell carcinomas were asked to participate in the study. The planned treatment was composite resection with microvascular soft tissue transfer (i.e., radial forearm free flap) for the reconstruction of surgical defects, and radiotherapy on indication. Exclusion criteria were age greater than 75 years, serious cognitive impairment and lack of basic fluency in the Dutch language. Twelve patients declined to participate, resulting in a final sample of 80 patients (response rate $=87 \%$ ). All patients were treated at the Department of Otolaryngology/Head and Neck Surgery of the VU University Medical Center, Amsterdam, The Netherlands.

\section{Data collection and study measures}

All data were collected prior to the start of treatment, including sociodemographics (age and gender), disease stage, comorbidity, HRQOL, and functional status. Comorbidity was assessed dichotomously (yes or no) by review of medical records and on the basis of selfreport, and was noted if one or more relevant medical ailments accompanied the primary medical illness. The comorbid conditions were cardiovascular, respiratory, gastro-intestinal, renal, endocrine, neurological, and immunological disorders, previous malignancy and considerable weight loss or alcohol abuse. For example, cardiovascular problems such as a myocardial infarct or hypertension, respiratory problems such as restrictive lung disease or COPD, or endocrine disorders such as diabetes mellitus with insulin usage were defined as relevant comorbid conditions.

HRQOL was assessed by means of the European Organization for Research and Treatment of Cancer (EORTC) QLQ-C30 questionnaire (version 2.0) [20] and the EORTC head and neck cancer module QLQH\&N35 [21]. The QLQ-C30 is composed of multi-item scales and single items assessing five areas of functioning (physical, role, emotional, cognitive, and social), fatigue, pain, emesis, dyspnea, insomnia, appetite loss, constipation, and diarrhea. Overall quality of life and the perceived financial impact of the disease and treatment are also assessed. The head and neck cancer-specific QLQ-H\&N35 module comprises seven symptom scales: pain, swallowing, senses, speech, social eating, social contact, and sexuality. There are 11 additional, single items covering problems with teeth, opening the mouth wide, dry mouth, sticky saliva, cough, feeling ill, weight loss, weight gain, use of nutritional supplements, feeding tubes, and painkillers. The scores of both the QLQ-C30 and of the QLQ-H\&N35 are linearly transformed to a scale of $0-100$, with a higher score indicating a higher (i.e., more positive) level of functioning or global HRQOL, or a higher (i.e., more negative) level of symptoms or problems.

The QLQ-C30 data of the patients were compared with published normative data from the general population of Norway [22]. Comparisons were made with the normative subsample that corresponded closely to the age and gender distribution of the patient sample (i.e., age range of $50-59$ years and $48 \%$ female). The QLQ-H\&N35 results were compared with reference values derived from a randomly selected sample of the Swedish general population [8].

Speech and oral functional status

Speech analyses were performed according to a standardized speech assessment protocol. Speech recordings of a read aloud text were performed in a soundtreated room and digitized using Cool Edit PRO 1.2 (Adobe Systems Incorporated, San Jose, CA, USA) with $22 \mathrm{kHz}$ sample frequency and 16 bit resolution. Recording level was adjusted for each speaker to optimize signal-to-noise ratio. All recordings were made with a mouth-to-microphone distance of $30 \mathrm{~cm}$. A computer program was developed to perform blinded randomized speech evaluation and to score overall 
intelligibility, and quality of articulation and nasal resonance. Overall intelligibility was assessed on a 10point scale ranging from poor to excellent by two trained speech therapists. Scores below six were defined as insufficient intelligibility (according to the Dutch educational system). To obtain more insight into the cause of decreased intelligibility, evaluation of the quality of articulation and nasal resonance was performed by the same two speech therapists on a 4-point scale ranging from deviant (score 1-3) to normal (score 4). Speech rate was measured by calculating words per minute on a read aloud standardized text.

Oral function was evaluated by a trained investigator (blinded for the clinical data regarding tumor site and stage) according to a protocol described by Teichgraeber et al. [23]. All assessments were based on 5point scales ranging from poor to excellent (transformed scores ranging from $0-100$ ). The oral function evaluation included three tests; (1) tongue mobility (mean score of tongue straight out, elevation of tongue tip, elevation of the base of the tongue, tongue deviation left and right, symmetry left and right, and tongue withdrawal), (2) lip mobility (mean score of general movement, spreading and rounding, symmetry left/ right, lip closure without speech, and lip closure during speaking), and (3) diadochokinesis (mean score of repetitive motion ability concerning tongue movement left/right, up/down, and in/out, and repeating the sylla$\mathrm{bles} / \mathrm{ta} / / \mathrm{cha} /$, and $/ \mathrm{ka} /$ ). Tongue and lip strength were quantified by a calibrated digital voltameter. To measure tongue strength the patient was asked to push the tongue against a metallic disc with the lips positioned around a cylinder, and to resist the force. To measure lip strength, the patient was asked to keep his lips around a button with a string attached to it on which the examiner pulled. Scores ranged from 0 (no strength) to $0.5 \mathrm{mV}$ (normal strength).

\section{Statistical analyses}

Chi-square tests were used to assess associations between the independent variables tumor site, classification and comorbid condition. Student's $t$-tests were performed to test for differences between the patient sample and the normative samples. These tests were based on the mean scores of the study and normative samples and the standard deviations of the study sample only, because no standard deviations of normative samples were available. Student's $t$-tests (HRQOL) and Mann-Whitney tests (functional status) were performed to determine the impact of tumor site (oral versus oropharyngeal), tumor classification (T2 versus T3T4) and comorbidity (yes or no). Spearman correlation coefficients $(r)$ were calculated to investigate the association between self-reported HRQOL (i.e., the QLQC30 global quality of life scale and the QLQ-H\&N35 speech scale) and speech and oral functional status. Statistical significance was defined as a $P$-value less than or equal to 0.05 .

\section{Results}

\section{Sample description}

Patient characteristics are shown in Table 1. The patients' age ranged from 23 to 74 years (mean 58 years). Forty-one percent of the sample was female. One patient was operated on for a recurrent tumor after prior transoral excision (1 year earlier) in which the rTNM stage was used, and one patient had undergone previous radiotherapy ( 3 years earlier) for a neck node of an unknown primary. Four patients had a synchronous second primary tumor, and in these cases the stage of the largest tumor was used. An equal percentage $(50 \%)$ of patients had a tumor originating on the left or right side, and in the majority of patients $(73 \%)$ the tumor did not extend over the median line. All 80 patients completed the EORTC questionnaires, speech rate tests (word count) and oral function tests (mobility and strength). In one patient speech recording could not be done due to logistical problems, and in three patients speech recordings were inadequate for interpretation due to technical problems. Speech quality analyses could thus be performed on 76 patients. Presence of comorbid conditions and tumor classification were significantly associated. Significantly more patients with comorbidity were diagnosed with a larger tumor $\left(\chi^{2}=10.37, P<0.01\right)$. No other significant associations were observed between tumor site, tumor classification, age, gender and comorbidity.

Health-related quality of life

Compared to the general population, the patients with oral or oropharyngeal cancer scored significantly worse on 5 (out of 15) scales or single items regarding the QLQ-C30: role functioning $(P=0.000)$, emotional functioning $(P=0.000)$, pain $(P=0.026)$, insomnia $(P=0.016)$ and appetite loss $(P=0.003)$. Conversely, patients scored significantly better regarding social functioning $(P=0.009)$, fatigue $(P=0.030)$, emesis $(P=0.001)$, and diarrhea $(P=0.001)$. Regarding the QLQ-H\&N35, the patients scored significantly worse on 7 (out of 10) scales or single items compared to the general population: pain $(P=0.000)$, swallowing 
Table 1 Characteristics of 80 patients included in this study

\begin{tabular}{ll}
\hline Age (years) & \\
Range & $23-74$ \\
Mean & 58 \\
& $n(\%)$ \\
Gender & \\
Male & $47(59)$ \\
Female & $33(41)$ \\
General condition & \\
Comorbidity & $48(60)$ \\
No comorbidity & $32(40)$ \\
Tumour site & \\
Oral cavity & $38(47)$ \\
Oropharynx & $42(53)$ \\
T & \\
2 & $35(44)$ \\
3 & $42(52)$ \\
4 & $3(4)$ \\
N & \\
0 & $24(30)$ \\
1 & $16(19)$ \\
$2 \mathrm{a}$ & $2(3)$ \\
$2 \mathrm{~b}$ & $30(38)$ \\
$2 \mathrm{c}$ & $6(7)$ \\
3 & $2(3)$ \\
\hline
\end{tabular}

$(P=0.000), \quad$ senses $\quad(P=0.050), \quad$ social eating $(P=0.000)$, teeth $(P=0.009)$, opening the mouth wide $(P=0.000)$ and sticky saliva $(P=0.005)$. Conversely, the patients scored significantly better on two items: coughing $(P=0.002)$ and feeling ill $(P=0.025)$.

Regarding tumor site, the QLQ-C30 results were comparable between oral cavity and oropharynx cancer patients. Only the pain score was significantly worse $(P=0.027)$ for patients with oral cavity tumors as compared to patients with oropharynx tumors. The QLQ-H\&N35 also revealed that patients with oral cavity tumors had significantly more pain $(P=0.002)$; furthermore, problems with their teeth appeared to be different $(P=0.001)$.

No statistically significant differences were observed for any of the QLQ-C30 scores as a function of tumor classification (T2 versus T3-T4). Regarding the QLQH\&N35, patients with T3-T4 tumors scored significantly worse on opening the mouth $(P=0.009)$ and reported feeling more ill $(P=0.035)$ compared to patients with $\mathrm{T} 2$ tumors.

Regarding comorbidity (Table 2), patients with one or more comorbid conditions scored significantly worse on QLQ-C30 physical functioning $(P=0.002)$, global quality of life $(P=0.016)$, fatigue $(P=0.014)$, pain $(P=0.024)$, constipation $(P=0.042)$ and diarrhea $(P=0.017)$ compared to patients without comorbidity. Additionally, based on the QLQ-H\&N35 data, patients with comorbidity reported significantly more pain $(P=0.007)$, trouble with social eating $(P=0.036)$,
Table 2 EORTC QLQ-C30 and QLQ-H\&N35 scores for comorbidity

\begin{tabular}{|c|c|c|c|}
\hline & \multicolumn{3}{|l|}{ Comorbidity } \\
\hline & \multirow{2}{*}{$\begin{array}{l}\text { No } \\
\text { Mean (SD) } \\
n=32\end{array}$} & \multicolumn{2}{|l|}{ Yes } \\
\hline & & $\begin{array}{l}\text { Mean (SD) } \\
n=48\end{array}$ & $P$ \\
\hline \multicolumn{4}{|l|}{ EORTC QLQ-C30 } \\
\hline Physical functioning & $95.0(12.4)$ & $81.7(21.4)$ & 0.002 \\
\hline Role functioning & $85.4(18.3)$ & $79.2(24.9)$ & 0.228 \\
\hline Cognitive functioning & $89.6(18.3)$ & $87.2(19.2)$ & 0.574 \\
\hline Emotional functioning & $69.5(23.4)$ & $72.2(24.6)$ & 0.626 \\
\hline Social functioning & $93.2(13.3)$ & $88.5(21.5)$ & 0.275 \\
\hline Global quality of life & $82.6(19.6)$ & $71.5(19.7)$ & 0.016 \\
\hline Fatigue & $13.9(20.0)$ & $26.9(24.1)$ & 0.014 \\
\hline Emesis & $1.6(4.9)$ & $1.4(4.7)$ & 0.874 \\
\hline Pain & $22.4(25.3)$ & $37.8(31.8)$ & 0.024 \\
\hline Dyspnea & $6.3(19.7)$ & $10.4(18.4)$ & 0.338 \\
\hline Insomnia & $36.5(36.3)$ & $31.3(39.1)$ & 0.550 \\
\hline Appetite loss & $7.3(20.3)$ & $18.7(31.4)$ & 0.072 \\
\hline Constipation & $5.2(20.9)$ & $18.7(32.9)$ & 0.042 \\
\hline Diarrhea & $1.0(5.9)$ & $8.3(16.1)$ & 0.017 \\
\hline Financial impact & $2.1(8.2)$ & $7.6(20.9)$ & 0.157 \\
\hline \multicolumn{4}{|l|}{ EORTC QLQ-H\&N35 } \\
\hline Pain & $27.1(17.6)$ & $41.5(25.5)$ & 0.007 \\
\hline Swallowing & $14.6(22.6)$ & $24.1(22.6)$ & 0.068 \\
\hline Senses & $6.3(14.5)$ & $8.7(21.5)$ & 0.577 \\
\hline Speech & $9.4(14.1)$ & $13.0(17.5)$ & 0.336 \\
\hline Social eating & $14.6(25.3)$ & $27.4(27.1)$ & 0.036 \\
\hline Social contact & $3.1(7.0)$ & $3.7(6.1)$ & 0.674 \\
\hline Sexuality & $16.1(24.9)$ & $16.3(27.6)$ & 0.981 \\
\hline Teeth & $13.5(26.6)$ & $30.6(38.8)$ & 0.034 \\
\hline Opening mouth & $7.3(20.3)$ & $25.7(37.2)$ & 0.013 \\
\hline Dry mouth & $15.6(26.8)$ & $22.2(28.6)$ & 0.303 \\
\hline Sticky saliva & $12.5(29.0)$ & $21.5(31.1)$ & 0.196 \\
\hline Coughing & $6.3(13.2)$ & $14.6(21.6)$ & 0.055 \\
\hline \multirow[t]{2}{*}{ Feeling ill } & $0(0)$ & $9.7(18.1)$ & 0.003 \\
\hline & $\%$ Yes & $\%$ Yes & \\
\hline Pain medication & 50.0 & 75.0 & 0.022 \\
\hline
\end{tabular}

teeth problems $(P=0.034)$, problems with opening the mouth $(P=0.013)$ and feeling ill $(P=0.003)$. The use of pain medication was significantly higher among patients with than those without comorbidity ( 75 versus $50 \% ; P=0.025)$.

Speech and oral functional status

Abnormal scores were observed in $17 \%$ of the patients regarding overall intelligibility, in $25 \%$ regarding nasality and in $37 \%$ regarding articulation. Functional results in relation to tumor site, classification and comorbidity are shown in Table 3. Regarding tumor site, patients with oral cavity tumors scored significantly worse on intelligibility $(P=0.015)$, articulation $(P=0.039)$, nasality $(P=0.040)$, tongue and lip mobility $(P=0.000,0.009)$, diadochokinesis $(P=0.004)$, and tongue strength $(P=0.001)$ compared to patients with 
Table 3 Functional status tests for tumour site, tumour stage and comorbidity

\begin{tabular}{|c|c|c|c|c|c|c|c|c|c|}
\hline & \multicolumn{3}{|l|}{ Tumour site } & \multicolumn{3}{|c|}{ Tumour stage } & \multicolumn{3}{|l|}{ Comorbidity } \\
\hline & Oral cavity & Oropharynx & & $\mathrm{T} 2$ & T3-T4 & & No & Yes & \\
\hline & $\begin{array}{l}\text { Mean (SD) } \\
n=37\end{array}$ & $\begin{array}{l}\text { Mean (SD) } \\
n=39\end{array}$ & $P$ & $\begin{array}{l}\text { Mean (SD) } \\
n=32\end{array}$ & $\begin{array}{l}\text { Mean (SD) } \\
n=44\end{array}$ & $P$ & $\begin{array}{l}\text { Mean (SD) } \\
n=29\end{array}$ & $\begin{array}{l}\text { Mean (SD) } \\
n=47\end{array}$ & $P$ \\
\hline \multicolumn{10}{|l|}{ Speech } \\
\hline $\begin{array}{l}\text { Intelligibility } \\
(0-10) \\
\text { Articulation }\end{array}$ & $6.0(1.3)$ & $6.7(1.0)$ & 0.015 & $6.8(1.0)$ & $6.0(1.2)$ & 0.011 & $6.6(1.0)$ & $6.2(1.3)$ & 0.132 \\
\hline$(0-4)$ & $3.4(0.8)$ & $3.7(0.6)$ & 0.039 & $3.8(0.4)$ & $3.3(0.8)$ & 0.003 & $3.8(0.4)$ & $3.4(0.8)$ & 0.043 \\
\hline $\begin{array}{l}\text { Nasality } \\
(0-4)\end{array}$ & $\begin{array}{l}3.6(0.6) \\
n=38\end{array}$ & $\begin{array}{l}3.8(0.4) \\
n=42\end{array}$ & 0.040 & $\begin{array}{l}3.8(0.4) \\
n=35\end{array}$ & $\begin{array}{l}3.7(0.6) \\
n=45\end{array}$ & 0.911 & $\begin{array}{l}3.7(0.4) \\
n=32\end{array}$ & $\begin{array}{l}3.7(0.5) \\
n=48\end{array}$ & 0.771 \\
\hline \multicolumn{10}{|l|}{ Rate } \\
\hline Words per minute & $\begin{array}{l}183(31.6) \\
n=38\end{array}$ & $\begin{array}{l}182(38.5) \\
n=42\end{array}$ & 0.988 & $\begin{array}{l}189(33.8) \\
n=35\end{array}$ & $\begin{array}{l}178(35.8) \\
n=45\end{array}$ & 0.201 & $\begin{array}{l}189(31.4) \\
n=32\end{array}$ & $\begin{array}{l}179(38.1) \\
n=48\end{array}$ & 0.233 \\
\hline \multicolumn{10}{|l|}{ Oral functions } \\
\hline Tongue (0-100) & $82.3(20.0)$ & $96.4(5.4)$ & 0.000 & $96.0(5.8)$ & $84.8(19.3)$ & 0.001 & $93.9(9.9)$ & $89.9(14.4)$ & 0.209 \\
\hline Lip $(0-100)$ & $87.4(5.7)$ & $99.7(0.9)$ & 0.009 & $99.4(2.0)$ & $98.0(5.1)$ & 0.140 & $99.3(2.2)$ & $98.5(3.9)$ & 0.239 \\
\hline Diadoch (0-100) & $87.4(20.7)$ & $97.2(6.1)$ & 0.004 & $97.5(5.0)$ & $88.7(19.6)$ & 0.012 & $97.4(5.1)$ & $91.5(15.4)$ & 0.026 \\
\hline Strength & & & & & & & & & \\
\hline Tongue $(0-0.5)$ & $0.2(0.2)$ & $0.4(0.2)$ & 0.001 & $0.4(0.2)$ & $0.3(0.2)$ & 0.021 & $0.4(0.2)$ & $0.2(0.2)$ & 0.045 \\
\hline $\operatorname{Lip}(0-0.5)$ & $0.3(0.1)$ & $0.4(0.2)$ & 0.328 & $0.4(0.2)$ & $0.3(0.1)$ & 0.018 & $0.4(0.2)$ & $0.3(0.1)$ & 0.370 \\
\hline
\end{tabular}

oropharyngeal tumors. Regarding tumor stage, patients with T3-T4 tumors scored significantly worse on intelligibility $(P=0.011)$, articulation $(P=0.003)$, tongue mobility $(P=0.001)$, diadochokinesis $(P=0.012)$, and tongue and lip strength $(P=0.021$, 0.018 ) than patients with T2 tumors. Patients with comorbidity scored significantly worse on articulation $(P=0.043)$, diadochokinesis $(P=0.026)$, and tongue strength $(P=0.045)$ as compared to patients without comorbidity.

Correlations between self-report and observer rated data

Significant correlations $(P<0.01)$ were observed between self-reported global quality of life scale (QLQ-C30) and observer ratings of intelligibility $(r=0.41)$ and articulation $(r=0.36)$ (Table 4). Additionally, statistically significant $(P<0.05)$ but relatively low correlations were found between self-reported speech (QLQ-H\&N35) and observer ratings of intelligibility $(r=-0.28)$, articulation $(r=-0.24)$, nasality $(r=-0.27)$ and diadochokinesis $(r=-0.28)$.

\section{Discussion}

Pretreatment HRQOL and functional status were investigated in a well-defined sample of patients with advanced oral and oropharyngeal cancer. Deteriorated
HRQOL and functional status before treatment have been reported in earlier studies, albeit for less well defined patient groups [9-15]. The results of the present study indicate a wide range of HRQOL and functional deficits in patients with advanced oral and oropharyngeal cancer before treatment. Impaired speech and oral function, as assessed objectively, was associated significantly with self-reported global HRQOL and speech problems, which is in accordance with the conclusions of Rogers et al. and Karnell et al. [14, 24].

Compared to the general population, patients scored significantly worse on 5 of 15 scales or items of the EORTC QLQ-C30 but, conversely, they scored significantly better on four others. Regarding the QLQ-H\&N35, patients scored significantly worse on most scales or items but significantly better on coughing and feeling ill. The significantly better scores for patients compared to the reference groups on some scales or items may be explained by the fact that people from the general population may have (other) chronic conditions as well. Alonso et al. [25] reported that, $55 \%$ of the general population has one chronic health condition, and $30 \%$ has more than one chronic condition. They also found that comorbidity can have a substantial impact on HRQOL, and stated that the presence of comorbidity limits the ability to attribute HRQOL deficits to one specific disease (e.g., to head and neck cancer).

In head and neck cancer patients, comorbidity has proven to be an important factor associated with 
Table 4 Correlations between functional status tests and QLQ-C30 global quality of life scale/QLQ-H\&N35 speech problems

\begin{tabular}{|c|c|c|c|c|}
\hline & \multicolumn{4}{|l|}{ Scales } \\
\hline & \multicolumn{2}{|l|}{ QLQ-C30 global quality of life } & \multicolumn{2}{|l|}{ QLQ-H\&N35 speech problems } \\
\hline & Correlation coefficient $(r) n=76$ & $P$ & Correlation coefficient $(r) n=76$ & $P$ \\
\hline \multicolumn{5}{|l|}{ Speech } \\
\hline Intelligibility & 0.411 & 0.000 & -0.285 & 0.013 \\
\hline Articulation & 0.355 & 0.002 & -0.242 & $\mathbf{0 . 0 3 5}$ \\
\hline Nasality & $\begin{array}{l}0.152 \\
n=80\end{array}$ & 0.190 & $\begin{array}{l}-0.270 \\
n=80\end{array}$ & 0.019 \\
\hline Rate & $\begin{array}{l}0.129 \\
n=80\end{array}$ & 0.267 & $\begin{array}{l}-0.142 \\
n=80\end{array}$ & 0.221 \\
\hline \multicolumn{5}{|l|}{ Oral functions } \\
\hline $\begin{array}{l}\text { Tongue } \\
\text { Lip } \\
\text { Diadochokinesis }\end{array}$ & $\begin{array}{l}0.078 \\
0.157 \\
0.109\end{array}$ & $\begin{array}{l}0.490 \\
0.164 \\
0.337\end{array}$ & $\begin{array}{l}-0.188 \\
-0.176 \\
-0.280\end{array}$ & $\begin{array}{l}0.095 \\
0.119 \\
\mathbf{0 . 0 1 2}\end{array}$ \\
\hline \multicolumn{5}{|l|}{ Strength } \\
\hline Tongue & 0.188 & 0.095 & -0.175 & 0.121 \\
\hline Lip & 0.056 & 0.624 & -0.049 & 0.665 \\
\hline
\end{tabular}

complications and mortality rates [26-29]. Studies on the impact of comorbidity on functional status in head and neck cancer patients are lacking. Studies that have examined the impact of comorbid status on HRQOL are scarce, have yielded conflicting results, and have examined the post-treatment period only. Pourel et al. [17] found no significant association between comorbidity and HRQOL in 113 long-term survivors 2 years after treatment for oropharyngeal carcinomas. Similarly, Taylor et al. [30] found no impact of comorbidity on work-related disability among 384 patients after treatment for head and neck cancer. However, Terrel et al. [19] in a study on HRQOL of 570 head and neck cancer patients after treatment, reported a clear effect of comorbidity in patients with two or more comorbid conditions. In our study on patients with oral or oropharyngeal carcinomas before treatment, comorbidity was present in $60 \%$ of the patients, and proved to have a major impact on HRQOL and functional status. Patients with comorbidity had significantly worse scores on several general and head and neck specific quality of life aspects and on speech and oral function tests compared to patients without comorbidity. Unfortunately, the cause of comorbidity was not systematically noted in the present study; future studies may provide more insight into the relation between the cause of comorbidity and HRQOL.

The impact of tumor site and classification on HRQOL appeared to be limited, with patients with oral cavity tumors reporting more pain, and patients with T3-T4 tumors reporting more trouble opening the mouth and feeling more ill. With regard to tumor site, comparisons with earlier studies are difficult because most studies included patients with oral cavity tumors only or reported on heterogeneous samples not stratified by (sub)sites [4, 9-11]. Regarding tumor classification, other studies have reported worse HRQOL for patients with higher tumor classifications and stages before treatment $[2,14]$.

Tumor site and classification were found to have a clear impact on functional status. Patients with oral cavity tumors (versus oropharyngeal tumors) and patients with T3-T4 tumors (versus T2 tumors) had worse speech and oral function scores, which is in accordance with the results of earlier studies [13,14].

In conclusion, we observed compromised HRQOL and functional deficits among patients with advanced oral and oropharyngeal cancer before the start of treatment. In addition to the impact of tumor site and classification, comorbidity proved to have a major impact on HRQOL and functional status. Prospective studies are needed to obtain insight into the relation between pretreatment HRQOL and functional status and outcome after treatment, and the relationship between changes in HRQOL and functioning over time and tumor site, tumor classification, and comorbid conditions.

Acknowledgments The authors would like to thank Jolijn Brouwer, MD, for her assistance with the collection of data, and Marike Koster, SLP, for performing the speech evaluation.

\section{References}

1. List MA, Lee Rutherford J, Stracks J et al (2002) An exploration of the pretreatment coping strategies of patients with carcinoma of the head and neck. Cancer 95:98-104 
2. Hammerlid E, Bjordal K, Ahlner-Elmqvist M et al (2001) A prospective study of quality of life in head and neck cancer patients. Part I: at diagnosis. Laryngoscope 111:669-680

3. Funk GF, Karnell LH, Dawson CJ et al (1997) Baseline and post-treatment assessment of the general health status of head and neck cancer patients compared with United States population norms. Head Neck 19:675-683

4. de Graeff A, de Leeuw JR, Ros WJ et al (2000) Pretreatment factors predicting quality of life after treatment for head and neck cancer. Head Neck 22:398-407

5. Bjordal K, Ahlner-Elmqvist M, Hammerlid E et al (2001) A prospective study of quality of life in head and neck cancer patients. Part II: Longitudinal data. Laryngoscope 111:14401452

6. McKinstry A, Perry A (2003) Evaluation of speech in people with head and neck cancer: a pilot study. Int J Lang Commun Disord 38:31-46

7. Fung K, Terrell JE (2004) Outcomes research in head and neck cancer. ORL J Otorhinolaryngol Relat Spec 66:207213

8. Hammerlid E, Taft C (2001) Health-related quality of life in long-term head and neck cancer survivors: a comparison with general population norms. Br J Cancer 84:149-156

9. Schliephake H, Jamil MU (2002) Prospective evaluation of quality of life after oncologic surgery for oral cancer. Int $\mathbf{J}$ Oral Maxillofac Surg 31:427-433

10. Kessler PA, Bloch-Birkholz A, Leher A et al (2004) Evaluation of quality of life of patients with oral squamous cell carcinoma. Comparison of two treatment protocols in a prospective study. Radiother Oncol 70:275-282

11. Shepherd KL, Fisher SE (2004) Prospective evaluation of quality of life in patients with oral and oropharyngeal cancer: from diagnosis to three months post-treatment. Oral Oncol 40:751-757

12. Schonweiler R, Altenbernd C, Schmelzeisen R et al (1996) Artikulationsfähigkeit und verständlichkeit der sprache bei patienten mit mundhöhlenkarzinomen. Ein vergleich präund postoperativer ergebnisse bei verschiedenen rekonstruktionstechniken. HNO 44:634-639

13. Colangelo LA, Logemann JA, Pauloski BR et al (1996) T stage and functional outcome in oral and oropharyngeal cancer patients. Head Neck 18:259-268

14. Rogers SN, Lowe D, Fisher SE et al (2002) Health-related quality of life and clinical function after primary surgery for oral cancer. Br J Oral Maxillofac Surg 40:11-18

15. Seikaly H, Rieger J, Wolfaardt J et al (2003) Functional outcomes after primary oropharyngeal cancer resection and reconstruction with the radial forearm free flap. Laryngoscope 113:897-904

16. Piccirillo JF (2000) Importance of comorbidity in head and neck cancer. Laryngoscope 110:593-602
17. Pourel N, Peiffert D, Lartigau E et al (2002) Quality of life in long-term survivors of oropharynx carcinoma. Int J Radiat Oncol Biol Phys 54:742-751

18. Fang FM, Chiu HC, Kuo WR et al (2002) Health-related quality of life for nasopharyngeal carcinoma patients with cancer-free survival after treatment. Int J Radiat Oncol Biol Phys 53:959-968

19. Terrell JE, Ronis DL, Fowler KE et al (2004) Clinical predictors of quality of life in patients with head and neck cancer. Arch Otolaryngol Head Neck Surg 130:401-408

20. Aaronson NK, Ahmedzai S, Bergman B et al (1993) The European organization for research and treatment of cancer QLQ-C30: a quality-of-life instrument for use in international clinical trials in oncology. J Natl Cancer Inst 85:365-376

21. Bjordal K, Hammerlid E, Ahlner-Elmqvist M et al (1999) Quality of life in head and neck cancer patients: validation of the European organization for research and treatment of cancer quality of life questionnaire-H\&N35. J Clin Oncol 17:1008-1019

22. Hjermstad MJ, Fayers PM, Bjordal K et al (1998) Using reference data on quality of life-the importance of adjusting for age and gender, exemplified by the EORTC QLQ-C30 (+3). Eur J Cancer 34:1381-1389

23. Teichgraeber J, Bowman J, Goepfert H (1986) Functional analysis of treatment of oral cavity cancer. Arch Otolaryngol Head Neck Surg 112:959-965

24. Karnell LH, Funk GF, Hoffman HT (2000) Assessing head and neck cancer patient outcome domains. Head Neck 22:6-11

25. Alonso J, Ferrer M, Gandek B et al (2004) Health-related quality of life associated with chronic conditions in eight countries: results from the international quality of life assessment (IQOLA) project. Qual Life Res 13:283-298

26. Borggreven PA, Kuik DJ, Quak JJ et al (2003) Comorbid condition as a prognostic factor for complications in major surgery of the oral cavity and oropharynx with microvascular soft tissue reconstruction. Head Neck 25:808-815

27. Borggreven PA, Kuik DJ, Langendijk JA et al (2005) Severe comorbidity negatively influences prognosis in patients with oral and oropharyngeal cancer after surgical treatment with microvascular reconstruction. Oral Oncol 41:358-364

28. Ferrier MB, Spuesens EB, Le Cessie S et al (2005) Comorbidity as a major risk factor for mortality and complications in head and neck surgery. Arch Otolaryngol Head Neck Surg 131:27-32

29. Derks W, de Leeuw RJ, Hordijk GJ (2005) Elderly patients with head and neck cancer: the influence of comorbidity on choice of therapy, complication rate, and survival. Curr Opin Otolaryngol Head Neck Surg 13:92-96

30. Taylor JC, Terrell JE, Ronis DL et al (2004) Disability in patients with head and neck cancer. Arch Otolaryngol Head Neck Surg 130:764-769 